\title{
Recenti Progressi dell'Archeologia ebraica in Italia ${ }^{(*)}$
}

\author{
Micaela Vitale
}

Dei numerosi insediamenti ebraici che nell'antichità si diffusero intorno al bacino del Mediterraneo solo quello di Roma, come noto, puó vantare il primato di persistenza ininterrotta dalla metà del II secolo a.C. fino ai giorni nostri.

In ordine alle numerose testimonianze letterarie pervenuteci sulle prime comunità ebraiche in Italia, va osservato che le fonti greco-latine in merito denunciano come già nell'antichità $i$ «gentili» nutrissero sentimenti contrastanti nei confronti di un popolo che si differenziava dalla cultura egemone locale: la curiosità per il "diverso" spesso si accompagna a commenti ironici o alla derisione e a volte traspare anche il disprezzo più totale. Sul versante opposto, $i$ testi di letteratura rabbinica ci forniscono notizie certo esenti da pregiudizi ma molto frammentarie.

In relazione a ció, si comprenderà bene quale importanza rivesta l'avvio, nella seconda metà degli anni ' 80 , della traduzione in italiano della monumentale opera di Emil Schürer, che fornirà ad un più vasto pubblico gli strumenti indispensabili per un corretto approccio allo studio degli Ebrei nell'antichità ${ }^{\text {. }}$.

Un ulteriore contributo alla migliore conoscenza delle prime comunità in Italia è poi fornito dalle sinagoghe e dalle catacombe, di cui ci sono rimaste vestigia ${ }^{2}$. Questi complessi archeologici -al di là del loro valore

Comunicación presentada en el Encuentro Internacional de Historiadores «En torno a Sefarad". Toledo 1991.

Schürer, E., La storia del popolo giudaico al tempo di Gesù Cristo, vol I, a cura di O. Soffritti. Brescia 1985; vol. Il, a cura di B. Chiesa. Brescia 1987. Traduzione della versione inglese, riveduta e corretta, a cura di G. Vermes, F. Miller e M. Black (Edimburgh 1973).

2 Si ricorda che sono note due sinagoghe: quella di Ostia, scoperta nel 1961, e quella di Bova Marina, rinvenuta nel 1985 e che rientra, quindi, nella presente relazione.

Per quanto riguarda i luoghi di sepoltura, si rammentano le sei catacombe di Roma, quella 
intrinseco- costituiscono anche il principale luogo di provenienza dei materiali mobili (per lo più epigrafi e lucerne) relativi agli Ebrei.

L'interesse per l'archeologia ebraica si è risvegliato a metà degli anni '70 allorquando, nel corso della trattativa per la revisione del concordato con la Chiesa cattolica (definita nel 1984), lo Stato italiano, facendo propria la richiesta dell'Unione delle Comunità Israelitiche Italiane (l'organismo rappresentativo dell'ebraismo italiano), preannunciava l'intendimento di acquisire la disponibilità delle catacombe ebraiche, da tempo custodite dalla Santa Sede, per poi concordare con l'Unione medesima la valorizzazione di tali monumenti.

A partir da quegli anni si registrano molteplici iniziative volte ad aggiornare le conoscenze in merito. E' nel 1976, infatti, che si pubblicarono i risultati della campagna di scavi condotta dalla Pontificia Commissione di Archeologia Sacra nelle catacombe di Villa Torlonia ${ }^{3}$.

Nel 1977 I'Unione prese contatti col Ministero dei Beni Culturali e con organismi scientifici stranieri allo scopo di fondare un comitato di patrocinio per le catacombe ebraiche, ma solo con la firma dell'Intesa fra lo Stato e l'Unione delle Comunità Ebraiche Italiane (U.C.E.I.) è stata ufficialmente prevista una commissione bilaterale per promuovere lo studio, la valorizzazione e la tutela del patrimonio cuturale ebraico ${ }^{4}$.

Se detti organismi a tutt'oggi non hanno ancora iniziato ad operare, maggiore successo è arriso ad un'iniziativa, patrocinata dall'Unione e conclusasi lo scorso anno, he ha coinvolto l'Amministrazione pubblica ed aziende private. Si tratta di una ricerca finnziata dallo Stato che, nell'arco di un biennio, si proponeva di censire e catalogare tutto il patrimonio culturale ebraico esistente in Italia ${ }^{5}$.

di Venosa in Basilicata e gli ipogei minori presenti in Sicilia (Noto e Siracusa) ed in Sardegna (S. Antioco).

3 FASOLA, U., "Le due catacombe ebraiche di Villa Torlonia", in Rivista di Archeologia Cristiana LII (1976), págs. 6-63.

${ }_{4}^{4}$ L'Intesa fu siglata nel febbraio 1987. L'art. 16, concernente $\mathrm{i}$ “Beni culturali ed ambientali» recita:

"Lo Stato, l'Unione e le Comunita collaborano per la tutela e la valorizzacione dei beni afferenti al patrimonio storico e artistico, culturale, ambientale e architettonico, archeologico, archivistico e librario dell'ebraismo italiano.

Entro 12 mesi dall'entrata in vigore della legge di approvazione della presente intesa sara costituita una Commissione mista per le finalita di cui al precedente comma e con lo scopo di agevolare la raccolta, il riordinamento e il godimento dei beni culturali ebraici.

La Commissione determina la modalita di partecipazione dell'Unione alla conservazione e alla gestione delle catacombe -ebraiche e le condizioni per il rispetto in esse delle prescrizione ritualli ebraiche. ma"

Alla medesima Commissione è data notizia del reperimento di beni di cui al primo com-

5 Il lavoro di catalogazione della presenza ebraica in Italia è stato reso possibile nell'ambito 
Il lavoro è stato svolto presso sedi dislocate sul territorio (oltre alla sede principale di Roma, si è operato ad Ancona, Bari, Firenze, Milano, Torino e Venezia coprendo pressochè tutto il territorio nazionale) e comprendeva oltre ad una fase preliminare di documentazione bibliografica ed archivistica, una di catalogazione vera e propria secondo i criteri dettati dall'Instituto Centrale per il Catalogo e la Documentazione (I.C.C.D.).

Tutte le schede prodotte sono informatizzate e, quanto prima, saranno consultabili presso il Centro Bibliografico dell'U.C.E.I., che ha sede a Roma.

Le ricerche condotte, se da un lato confermano ed avvalorano i dati $\mathrm{e}$ le notizie già conosciute, dall'altro hanno considerevolmente aumentato il bagaglio documentario relativo a siti dove finora la presenza ebraica non era riconosciuta o era solo parzialmente nota.

Per quanto riguarda il materiale archeologico - pero lo più proveniente dalle regioni centro-meridionali- il limite di due anni ha richiesto delle scelte metodologiche che hanno sacrificato, in genere, lo studio dei materiali non custoditi in collezioni pubbliche. Si è quindi giunti all'elaborazione di circa 250 schede comprendenti le iscrizioni funerarie, le lucerne con la rappresentazione della menorah e quelle comunque provenienti dal complesso della sinagoga di Ostia - oggetto di specifico studio-e, infine, le catacombe di Venosa in Basilicata.

Il tempo previsto per la catalogazione non è risultato sufficiente a completarla; era stato quindi richiesto un nuovo finanziamento che ne permettesse il proseguimento ma questo non è stato ancora concesso, pertanto il lavoro risulta ancora incompleto.

Vanno inoltre segnalate le varie iniziative promosse dagli studiosi in tema di storia dell'ebraismo.

Alla Settimana di Studi che si svolse a Spoleto nel 1978 ed ebbe per tema "Gli Ebrei nell'Alto medioevo" fece seguito, nel 1981, il I Congresso Internazionale "Italia Judaica", organizzato da una commissione mista composta da membri dell'Università di Ferrara, del Ministero dei Beni Culturali (Ufficio Centrale per i Beni archivistici), della Hebrew University di Gerusalemme e dell'Università di Tel Aviv ${ }^{6}$.

delia Legge 41/1986, c.d. Legge del "Giacimenti Culturali» e si è svolto principalmente nel corso del biennio 1988-89.

${ }^{6}$ Bari, 18-22 maggio 1981. Contestualmente, presso l'Archivio di Stato, venne allestita una piccola mostra di fonti documentarie ed archeologiche. 
In quella occasione C. Colafemmina, dell'Università di Bari, presentó un aggiornamento bibligrafico ed una ricerca, condotta tramite le lapidi funerarie, sulle tracce, spesso frammentarie e oltremodo disperse, della presenza ebraica nell'Italia merdionale dal IV secolo in avanti ?.

In quella stessa occasione, con una visita guidata, vennero presentate le catacombe di Venosa che, in collaborazione con l'università americana di Duke, erano in corso di studio ${ }^{8}$.

Altro momento di approfondimento e confronto è stato, nel 1987, il XVI incontro di studiosi dell'Antichità cristiana ${ }^{9}$ in cui F. Bisconti e L. Dequeker proposero una nuova lettura di due temi raffigurati nelle catacombe ebraiche di Roma. II primo dimostró una continuità iconografica nelle scene di "uomo circondato dagli animali», analizzate in contesti ideologici diversi fra loro (Apollo musageta, Orfeo citaredo, Davide salmista e Adamo-Buon Pastore). II secondo insistette sul valore simbolico-escatologico della rappresentazione dell'arca dell'alleanza, contrastando la teoria che vorrebbe vedere in questo motivo solamente l'attestazione di una fede religiosa ${ }^{10}$.

Da ultimo, lo scorso anno, I'VIII convegno sull'Africa Romana è stato ulteriore occasione di riflessione anche sull'antica diaspora ebraica nel bacino del Mediterraneo. In particolare, G. Marasco, attraverso un'accurata indagine delle fonti letterarie e di quelle critiche, ha analizzato le cause, lo svolgimento e le finalità del famoso esilio, decretato da Tiberio, di 4.000 ebrei in Sardegna ".

In un altro contesto, sono da segnalare i contributi di C. Vismara che ha pubblicato i risultati della sua analisi della bibliografia esistente in merito agli Ebrei dell'antica Roma, e più specificatamente alle catacombe a cui questi afferivano, e di una prima perlustrazione condotta all'interno di quella di Vigna Randanini sull'Appia antica. Si tratta sempre di articoli

Colafemmina, C., "Archeologia ed epigrafia ebraica nell'ttalia meridionale", in Italia Judaica I. Roma 1983, págs. 189-210, con tutta la documentazione raccolta e relativa bibliografia, sulla Campania, Puglia, Basilicata, Calabria, Sicilia (con Malta) e Sardegna.

- Colafemmina, C., "Nuove scoperte nella catacomba ebraica di Venosa", in Puglia Paleocristiana e Alto medievale IV. Bari 1984, págs. 33-49.

9 Cristianesimo e Giudaismo: eredita e confronti. Roma, 7-9 maggio 1987. Gli atti sono stati pubblicati in Augustinianum xxvIII, 1-2 (1988).

10 Rispettivamente: BISCONTI, F., «Un fen'omeno di continuita iconografica: Orfeo citaredo, Davide salmista, Cristo pastore, Adamo e gli animali», in Augustinianum, cit., págs. 429-436; DEQUEKER, L., "L'iconographie de l'arche de la Torah", ibidem, págs. 437-460.

1 Solin. H., "Gli Ebrei d'Africa: una nota", in Atti dell'VIll Convegno sull'Africa Romana. Cagliari - dicembre 1990, Sassari 1991, págs. 615-623; MARAsco. G., "Tiberio e l'esilio degli Ebrei in Sardegna", ibidem, págs. 649-659. 
"aperti», che offrono cioè una serie di spunti per le ricerche successive ${ }^{12}$.

Nel principale di questi articoli, dopo una sintesi storica d'inquadramento, l'autrice si concentra sulle notizie relative alle catacombe conosciute, soffermandosi maggiormente sui problemi posti da quelle di Vigna Randanini e di via Appia Pignatelli.

Per quanto concerne la prima, mai studiata approfonditamente, pone l'accento sui punti da sviluppare in sede di intervento. Ad esempio riguardo all'aula fuoriterra: la sua funzione originaria, il suo rapporto con l'ipogeo, il motivo della sua parziale trasformazione in ambiente funerario; oppure, in merito alla catacomba in senso stretto: il modo di sviluppo cronologico degli spazi e il rapporto tra i due accessi dal sopraterra e le varie regioni, la diversa disposizione dei tipi di sepoltura ${ }^{13}$ e quindi il più generale problema dell'organizzazione interna del cimitero. Questa è dettata dal censo o, come mi sembra più probabile, dal paese di origine?

Per quanto riguarda, invece, la catacomba di via Appia Pignatelli, si contestano tutte le motivazioni addotte dal suo scopritore (Müller) per considerarla ebraica. La più significativa obiezione è il rilevare la totale as. senza di simboli ebraici.

In tema di epigrafia ebraica, va menzionata la recente tesi di laurea di S. Frascati, "Le iscrizioni giudaiche latine di Roma. Revisione sistematica» ${ }^{14}$, nella quale, attraverso la revisione del Corpus Inscriptionum Latinarum VI, relativo alla città di Roma, si è giunti ad importanti conclusioni.

Innanzi tutto, eseguendo quando possibile un esame autoptico, la studiosa ha ottenuto un notevole successo riconoscendo, fra le iscrizione ritrovate da padre Fasola a Villa Torlonia, la parte sinistra di una iscrizione perduta anche se già pubblicata e riprodotta ${ }^{15}$.

12 Vismara, C., “l cimiteri ebraici di Roma», in Società romana e impero tardoantico, vol. II, Roma: politica economia e paesaggio urbano, a cura di A. Giardina. Roma-Bari 1986, págs. 351 392, 490-503 con le successive "note e discussioni" in "Ancora sugli Ebrei di Roma", Archeologia Classica xxxvil-XL (1986-1988), págs. 150-161 e “Orientali a Roma: nota sull'origine geografica degli Ebrei nell testimonianze di età imperiale» in Dialoghi di Archeologia $(1987,1)$, págs. 119-121.

${ }_{13}$ Si ricorda la presenza di quella forma di sepoltura detta Kokh (pl. Kokhim), impropriamente tradotto in "tomba a forno" dalla sua disposizione perpendicolare alla parete in cui è albergata, di origine orientale e di cui si hanno precedenti nelle necropoli ipogee di Beth Shearim in Israele e a Gamart in Tunisia; cfr. VITALE. M., "Le catacombe ebraiche: premesse per un recupero", La Bassegna Mensile di israel LII $(1986,1)$, págs. 176-186, ivi págs. 177-178.

14 Universitá degli Studi di Roma «La Sapienza", Cattedra di Epigrafia Latina, a.a. 1988/89.

15 Frascati, S., "Un'iscrizione giudaica dalle catacombe di Villa Torlonia», Rivista di Archeologia Cristiana LXV (1989), págs. 135-142. 
Un altro risultato di cui bisogna dar merito riguarda il problema dell'appartenenza alla religione ebraica di una serie di metuentes. Questo viene affrontato dimostrando definitivamente che il termine ricorre anche in epigrafi di ambiente pagano e che quindi, in caso di mancata specificazione, non debbano riferirsi necessariamente a dei convertiti all'ebraismo.

Nei limiti che una tesi di laurea in epigrafia impone, le problematiche più strettamente ebraiche non vengono adeguatamente sviluppate ma mi preme di suggerire come spunto di ulteriore verifiche, la possibilita che Alexander bubularus de macello fosse, in proprio o più facilmente incaricato da una Comunità, un macellatore rituale (shochet).

Nella tesi purtroppo si ribadisce l'impossiblità di maggiori specificazioni cronologiche ma si conferma che la datazione di tali reperti risale ai secoli III e IV della nostra era.

Fra le novità più sorprendenti di questi anni bisogna ricordare che nel 1985, a Bova Marina (provincia di Reggio Calabria), in modo del tutto fortuito, è stato scoperto un edificio che, per la presenza di un mosaico pavimentale con la rappresentazione di una menorah e di altri oggetti cultuali ebraici, si identifica sicuramente come sinagoga ${ }^{16}$.

L'area esplorata si trova alla periferia di un insediamento, verosimilmente una statio della strada costiera, sviluppatosi intorno ad una villa preesistente. Tale area risulta frequentata fin dal II secolo ma libera da strutture fino al IV, epoca in cui si collocano perfettamente la sequenza stratigrafica e lo stile del mosaico.

La pianta di questo complesso di vani non trova puntuali confronti con altri edifici sinagogali conosciuti ma non si discosta molto dalla struttura (ribaltata specularmente) della sinagoga di Ostia. Nella sua prima fase risulta essere di forma pressochè quadrata, divisa in due parti; una con due aule anch'esse quadrate, l'altra con tre vani paralleli disposti ortogonalmente alle prime. All'inizio del VI secolo, invece, bisogna datare alcune trasformazioni strutturali che ingrandiscono il complesso e dividono in maniera diversa le stanze rettangolari esistenti. Anche l'aula sinogogale vera e propria viene modificat con l'aggiunta di un'absidiola spórgente sul lato est.

16 LATTANZI, E., "Attivita' della Soprintendenza Archeologica della Calabria", Kleorchos 105109 (1985), págs. 134-136; idem, "Rassegna Archeologica Calabrese», Magna Grecia 21, 3-4 (1986), págs. 6-7; idem, Archeo 14 (aprile 1986), pág. 14. Recentemente una comunicazione scientifica e stata presentata da L. Costamagna nell'ambito del Seminari di Archeologia Cristiana che si svolgono presso la sede della Scuola Francese di Roma (14 noviembre 1991). 
Nei vari ambienti circostanti non sono state rinvenute tracce né del bagno rituale (migweh) né di un forno per le azzime, mentre si suppone l'identificazione della genizah (deposito nascosto degli oggetti sacri divenuti inutilizzabili) in un piccolo dolio interrato in un angolo dell'aula principale; risulta, invece, oscura la funzione di un dolio di grandi dimensioni, interrato in un'area del VI secolo ove è stato rinvenuto anche un tesoretto con oltre 3000 monete bronzee databili dal III al $\mathrm{V}$ secolo.

II complesso cessa bruscamente di vivere intorno alla fine del secolo e l'area non viene riutilizzata. Attualmente, dopo gli scavi svoltisi fino al 1987, questa è stata completamente reinterrata per permettere il proseguimento del lavori previsti per la costruzione della variante della superstrada Ionica.

Altre attività, non meno importanti ma rivolte ad un pubblico meno specializzato, sono le mostre che si sono svolte a Roma e a Ferrara.

$\mathrm{Nel} 1985$ è stata realizzata «Voltre della Memoria. Immagini ebraiche e cristiane nelle catacombe di Roma" ${ }^{17}$ in cui, attraverso immagini fotografiche e riproduzioni dal vero delle pitture e delle iscrizioni catacombali, si ribadiva il concetto dell'assorbimento della cultura iconografica pagana e la sua apposizione a tematiche bibliche. Questa commistione è stata più volte rilevata e documentata ma, per la prima volta, ha varcato gli intenti strettamente scientifici, per riunire in un breve catalogo schede e immagini divise schematicamente per tematiche generali.

Nel 1990, a Ferrara, città che si pone come polo culturale a quindi come sede adatta al recupero della cultura ebraica nazionale, si è invece svolta «I TAL YA - Isola della rugiada divina. 2000 anni di arte e di vita ebraica in Italia" ${ }^{18}$. Nel catalogo, come la mostra, trasposizione di quello americano, si segnala l'articolo di R. Brilliant ${ }^{19}$ utile per l'accostamento delle fonti letterarie romane e ebraiche ma in cui una fuorviante traduzione dell'inglese shrine in reliquiario - prendendo a prestito un termine tipico del cattolicesimo- inconsapevolmente rende l'ebraismo un qualcosa d'immutabile o forse morto.

Questa mostra è stata anche resa possibile grazie al prestito di alcuni reperti da parte della Soprintendenza Archeologica di Roma in attesa della

\footnotetext{
17 Roma, Castel S. Angelo - novembre 1985- curata da E. Shochet Brettman dell'International Catacomb Society di Boston (USA).

18 Ferrara, Palazzo dei Diamanti 18 marzo/17 giugno 1990, edizione italiana di aGardens and Ghettos" New York, Jewish Museum - 17 settembre 1989/1 febbraio 1990 a cura di V. B. Mann.

19 BRILLIANT, R., "Arte e cultura ebraiche nell'Italia antica", in / TAL YA. Milano 1990, págs 69-85.
} 
realizzazione di un'esposizione integrale del materiale in suo possesso e di sua competenza.

Sperando di non aver tralasciato contributi o attività importanti, pubblicati o svoltesi in Italia di recente, esprimo l'auspicio che i prossimi anni possano essere vieppiù fecondi per l'archeologia ebraica, anche senza dover contare sulle, pur sempre benvenute, scoperte. 